\title{
Ferroelectric Based Microgyroscope for Inertial Measurement Unit: Modeling and Simulation
}

\author{
Zafer Ozer \\ Mersin University \\ Mersin, Turkey \\ e-mail: zaferozer@hotmail.com
}

\author{
Amirullah M.Mamedov, Ekmel Ozbay \\ Bilkent University \\ Nanotechnology Research Center (NANOTAM) \\ Ankara, Turkey \\ e-mail: mamedov@bilkent.edu.tr
}

\begin{abstract}
This paper present the design and modeling of the micro-electromechanical systems (MEMS) on the ternary ferroelectric compounds (PZT and $\left.\mathrm{Ba}_{\mathrm{x}} \mathrm{Sr}_{1-\mathrm{x}} \mathrm{TiO}_{3}\right)$ based by using finite element model (FEM) simulation.
\end{abstract}

Keywords-MEMS, ferroelectrics, FEM

\section{INTRODUCTION}

Micro-Electro-Mechanical Systems (MEMS) is the integration of mechanical elements, sensors, actuators and electronics on a common silicon or other substrate through micro fabrication technology. While the electronics are fabricated using integrated circuit (IC) process sequences (e.g., CMOS, Bipolar or BICMOS processes), the micromechanical components are fabricated using compatible "micromachining" processes that selectively etch away parts of the silicon wafer or add new structural layers to from the mechanical and electromechanical devices.

MEMS gyroscopes rely on two principles. The first principle is the resonating vibration of proof mass. The second principle is the Coriolis effect [1-2].

The physical mechanisms underlying MEMS devices include capacitive, piezoresistive, electromagnetic, piezoelectric, ferroelectric, optical and tunneling. The most successful types are based on capacitive transduction [3-5].

In this paper, we design novel structures, which are resistant to shock and sensitive to biaxial applied angular velocity, and analyze different reference vibrations. Finally, the best one with higher resolution, lower coupling effect and convenient anchor is chosen as device. Also we analyze the operation principle, discuss the problem about fixing gyroscope and find a special point to detect resonance mode. This gyroscope has a simple structure and is completed solid. It is excited in special resonance mode and utilized mode vibration of piezoelectric materials (as material we used PZT-ceramics and $\mathrm{Ba}_{\mathrm{x}} \mathrm{Sr}_{1}$. ${ }_{x} \mathrm{TiO}_{3}$ ) as reference vibrations.

\section{MEMS GYROSCOPE STRUCTURE, DESING AND SIMULATION}

ANSYS is general-purpose finite element analysis (FEA) software package [6]. Finite Element Analysis is a numerical method of deconstructing a complex system into very small pieces (of user-designated size) called elements.

The software implements equations that govern the behavior of these elements and solves all, them of creating a comprehensive explanation of how the system acts as a whole.

This type of analysis is typically used for the design and optimization of a system far too complex to analyze by hand. Systems that may fit into this category are too complex due to their geometry, scale, or governing equations.
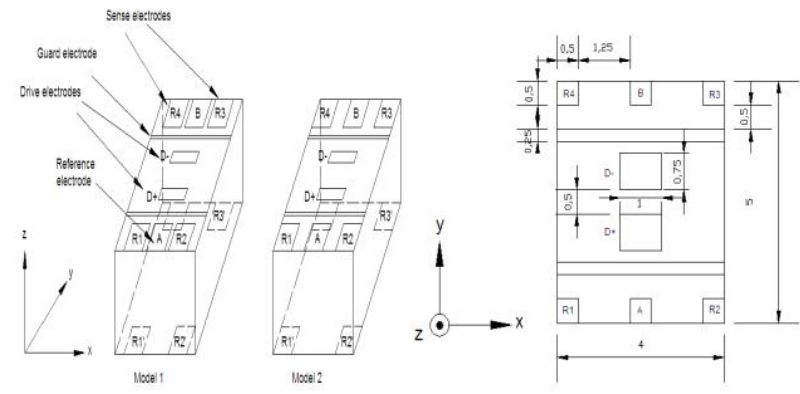

Figure 1. Principle of operation and detail structure of the solid state gyroscope

In 2006 Maenaka etc, proposed a novel piezoelectric solid gyroscope [7], which is named as piezoelectric micromachined modal gyroscope (PMMG) [8]. The basic operation principle of the device is shown in Figure 1. At first, assume that the rectangular prism is made of PZT, and it has polarization along $z$-axis. With excitation of the PZT prism at the operation mode mentioned before, the differential vibration to $x$-axis in the prism appears as shown in Figure 1. When the angular velocity along $y$-axis is applied, the Coriolis force is generated according to movement of the mass elements, resulting in compressive and tensile stresses depending on the position. These stresses differentially generate the piezoelectric voltage at the surface of the device. 
This voltage is proportional to the applied angular rate and is an output signal of the device. Piezoelectric ceramic material PZT is selected as the substrate block. On the top and bottom surfaces of the PZT block, a lot of driving electrodes and sensing electrodes are distributed. As shown in the figure, D+ and $\mathrm{D}^{-}$are the driving electrodes, and the exciting voltages are applied on the $\mathrm{D}^{+}$and $\mathrm{D}-$ electrodes. A and $\mathrm{B}$ are the reference electrodes, which can be used for searching and tracking the working resonance mode. R1, R2, R3, and R4 are the sensing electrodes. When there is no angular input, the voltages of two adjacent electrodes, such as R1 and R2, or R3 and R4, are the same because of the symmetric structure of the piezoelectric block. If the rotation in any direction perpendicular to the modal vibration is input, the voltage of sensing electrodes is changed because of the coupling of the Coriolis effect, and then the voltages of two adjacent electrodes are not the same. Through detecting the voltage difference of two adjacent sensing electrodes, the rotation input can be quantized. On the bottom surface, the sensing and driving electrodes are distributed in the corresponding positions as on the top surface.

\section{RESULT AND DISCUSSIONS}

\section{A. Material Selection.}

Piezoelectric material is commonly used for electromechanical transducers, and the requirements for the performance of piezoelectric ceramic vary for different regions of applications. As for the solid-state microgyroscope we used, the piezoelectric material serves as the excitation source and sensing element simultaneously, so the piezoelectric material should have larger piezoelectric constant $d_{33}$ and electromechanical coupling constants $k_{33}$ and $k_{15}$.

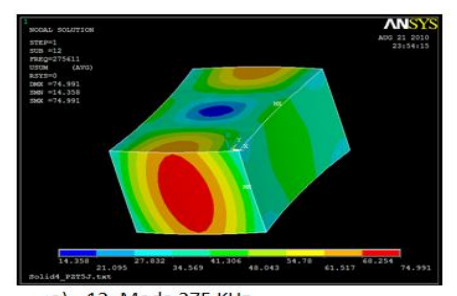

a) 12. Mode $275 \mathrm{KHz}$

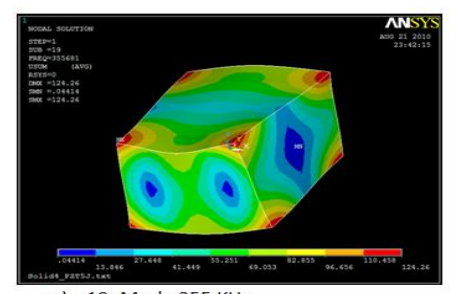

a) 19. Mode $355 \mathrm{KHz}$

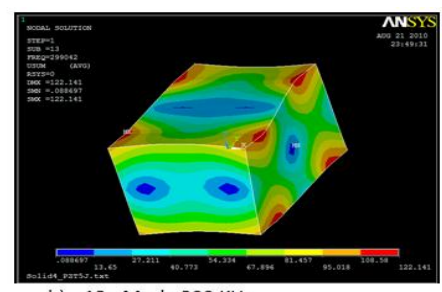

b) 13. Mode $299 \mathrm{KHz}$

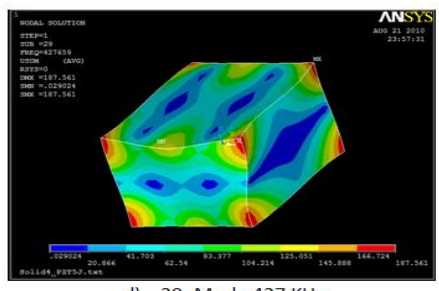

d) 29. Mode $427 \mathrm{KHz}$
Figure 2. Longitudinal resonance modes with no DOF restrictions. (PZT 5 and contours show displacement sum).
Based on these conditions, we selected PZT during following simulation. The PZT material constants used in this paper are listed as follows: the relative dielectric permittivity $\left(\varepsilon_{r}\right): \varepsilon_{11}=$ $2740, \varepsilon_{33}=1670$, material mass density $(\rho): 7400 \mathrm{~kg} / \mathrm{m}^{3}$, the piezoelectric constant $(e): e_{31}=-8.5 \mathrm{C} / \mathrm{m}^{2}, e_{33}=28.3 \mathrm{C} / \mathrm{m}^{2}, e_{15}$ $=20.0 \mathrm{C} / \mathrm{m}^{2}$, the elastic constant matrix $(c): c_{11}=13.6 \times 10^{10}$ $\mathrm{N} / \mathrm{m}^{2}, c_{12}=8.05 \times 10^{10} \mathrm{~N} / \mathrm{m}^{2}, c_{13}=8.72 \times 10^{10} \mathrm{~N} / \mathrm{m}^{2}, c_{33}=$ $12.4 \times 10^{10} \mathrm{~N} / \mathrm{m}^{2}, c_{44}=2.40 \times 10^{10} \mathrm{~N} / \mathrm{m}^{2}, c_{66}=2.411 \times 10^{10}$ $\mathrm{N} / \mathrm{m}^{2}$

\section{B. Modal Analysis}

In this section, the finite element analysis of the piezoelectric body of the PMMG was first conducted to find the operation mode, and then quantitative indicators were introduced to evaluate these modes and at last the best operation mode and the corresponding size of the device was given. From the operation principle of the PMMG, it can be concluded that the working resonance mode should have the following characteristics. I. The movement of points in the piezoelectric block should be almost in one direction, $y$-axis in this paper. II. The moving direction of points should be perpendicular to the polarization direction of the piezoelectric block. III. The moving direction of a point on one edge is the same as that of the corresponding point on the diagonal edge and is opposite to that of the corresponding point on the adjacent edge. IV. Moving edges should be in the state of tension or compression. To get this special modal shape, we used ANSYS to perform modal analysis and list corresponding frequencies under which the modal shapes meet these characteristics. The corresponding results are listed at Table.

\section{Harmonic Analysis.}

In real applications, we need to apply a driving voltage to actuate the device. To make sure the mode shape we activate is the same as the one we get in modal analysis, we used ANSYS to perform harmonic analysis. The configuration of the driving electrodes is shown in Figure 1. The amplitude of driving voltage is up to $10 \mathrm{~V}$, and the phase difference of the voltages on $\mathrm{D}+$ and $\mathrm{D}-$ is $180^{\circ}$. The damping constant of the piezoelectric material was introduced with the value of 0.02 . The frequency of the driving voltage was scanned from 200 $\mathrm{kHz}$ to $700 \mathrm{kHz}$. Figures 2 - 5 gives the harmonic analysis results of the PMMG. In Figure 4(a), the $x$-coordinate refers to the frequency of the driving voltage and the $y$-coordinate refers to the piezoelectric voltage amplitude on the reference electrode, R1 or R2. From the figure, we can see that there are two peaks of the voltage of the reference electrode. The peak corresponding to the working resonance mode is in the position of around $400 \mathrm{kHz}$. The frequency of the working resonance mode is $452251 \mathrm{~Hz}$. Figure 4(b) shows the mode shape at the exciting frequency of the working resonance mode. It is concluded that the exciting vibration of the piezoelectric block is the same as the vibrating shape of the working resonance mode. That is to say that through applying driving voltage with the frequency of the working resonance mode on the driving electrodes, the vibration of the working resonance mode can be actuated account of the Coriolis force. Because the raw result of the output voltage for the applied 

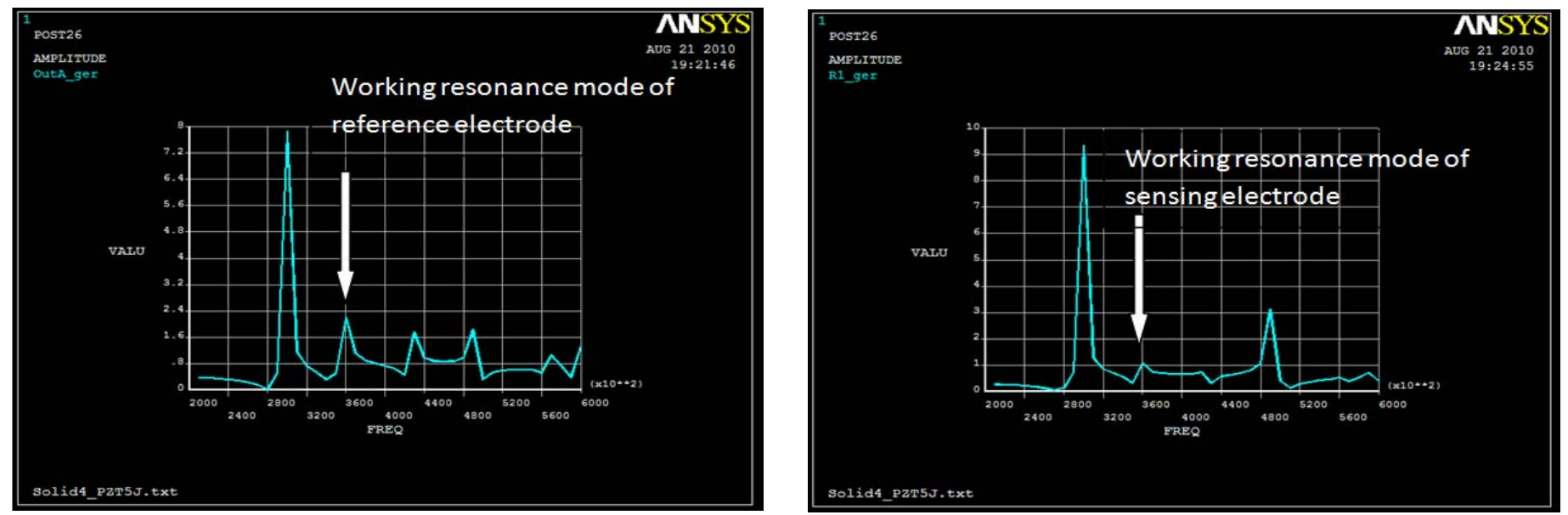

Figure 3. The piezoelectric voltage on reference electrode versus different driving frequencies.

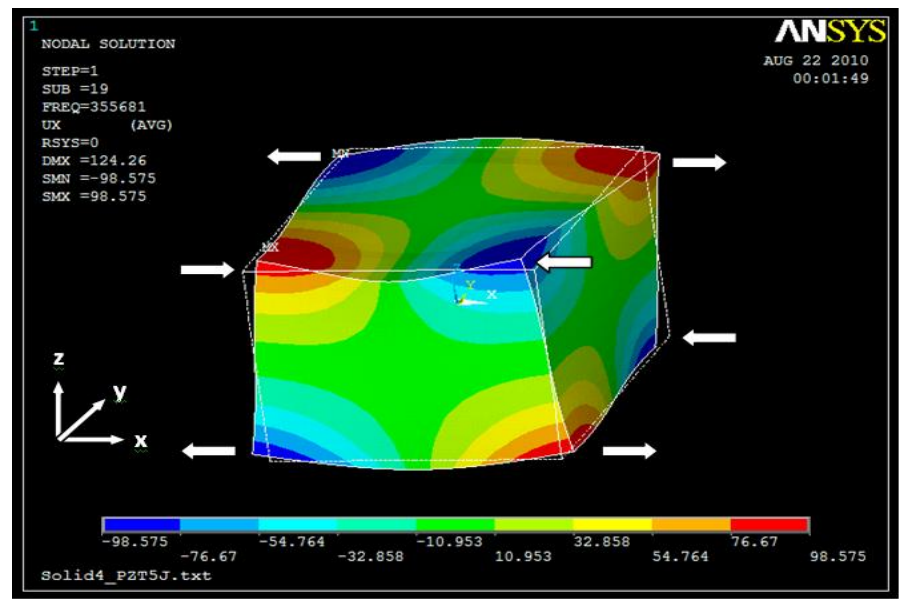

(a)

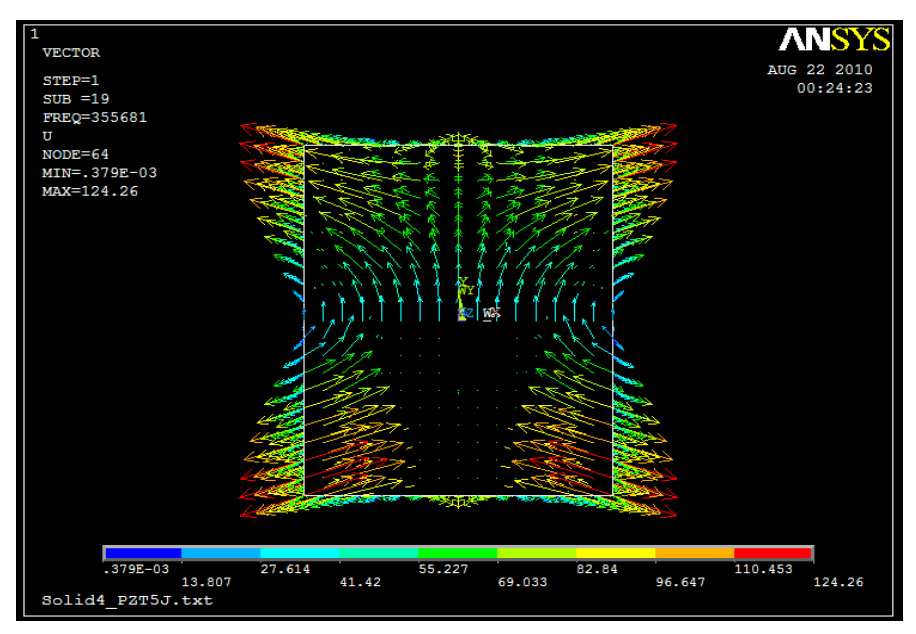

(b)

Figure 4. The x-axis displacement counter (a) and the mode displacement vectors (b) on top surface of the 19th mode.
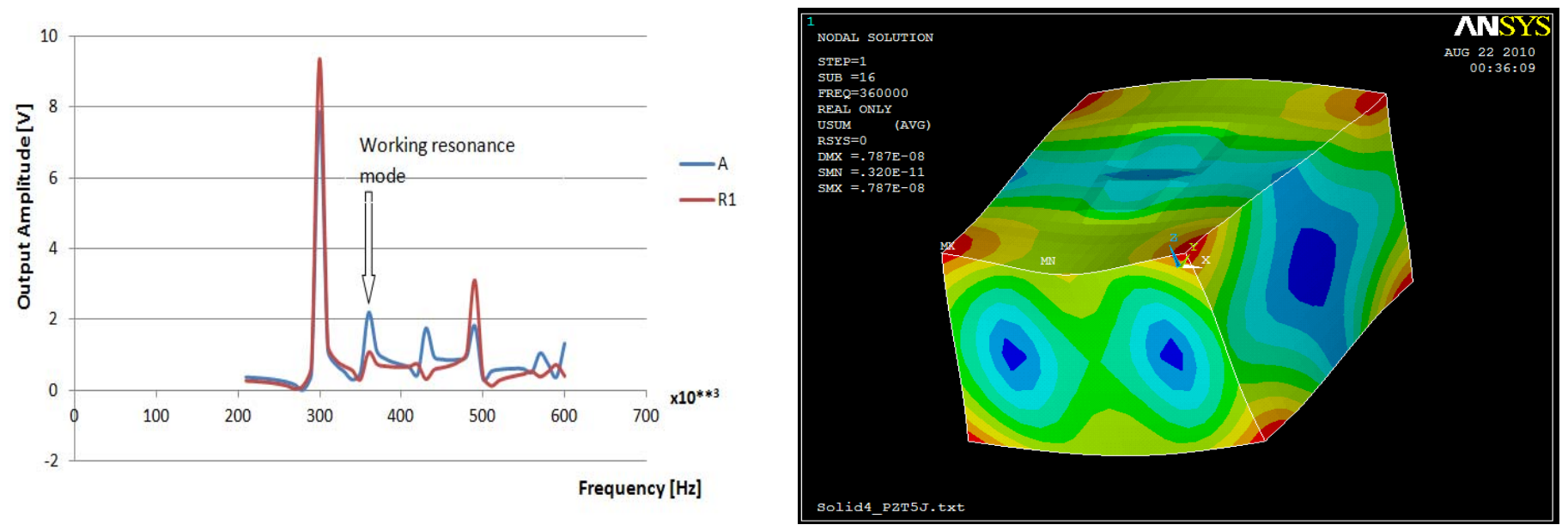

(a)

(b)

Figure 5. Harmonic excitation analysis result of the modal gyroscope. 
angular velocity is differential with respect to $x=0$, we applied subtraction of the potentials at symmetric points, that is, $V$ sub1 $=V \mathrm{D} 1-V \mathrm{D} 2$ and $V$ sub2 $=V \mathrm{D} 3-V \mathrm{D} 4$. Fig. 6 shows the configuration of the driving electrodes, and Fig. 6 show the relation between $V$ sub1, $V$ sub2 and angular velocity applied to the device, respectively. As we can see from Figure $6, V$ sub1 and $V$ sub2 have a liner relation with angular velocity, which agrees with the working principle of PMMG and testifies that the rotation input can be quantized through detecting the voltage difference of two adjacent sensing electrodes. of D2. To further optimize the size of the additional driving electrodes, we introduced three variables which should be changed: the length of the driving electrodes, the voltage applied on the driving electrodes, and the distance between two adjacent electrodes.

Table 1. The calculated performance of gyroscopes, when some resonance modes are used as reference vibration.

\begin{tabular}{|c|l|l|l|l|l|l|}
\hline & PZT4 & PZT5H & PZT5J & PZT6B & PZT7A & PZT8 \\
\hline $\begin{array}{c}\text { Working } \\
\text { Resonanace } \\
\text { Frequency } \\
\text { (Hz) }\end{array}$ & 403867 & 452251 & 355681 & 558485 & 429330 & 410531 \\
\hline $\begin{array}{c}\text { Sense axis } \\
\text { displacement } \\
\text { of A } \\
\text { electrocode } \\
\text { (e-8 m) }\end{array}$ & 2.79 & 1.67 & 3.70 & 5.69 & 1.41 & 1.37 \\
\hline $\begin{array}{c}\text { Sense axis } \\
\text { displacement } \\
\text { of RI } \\
\text { electrode (e- } \\
\text { 8 m) }\end{array}$ & 2.59 & 1.09 & 3.35 & 1.06 & 1.29 & 1.44 \\
\hline
\end{tabular}

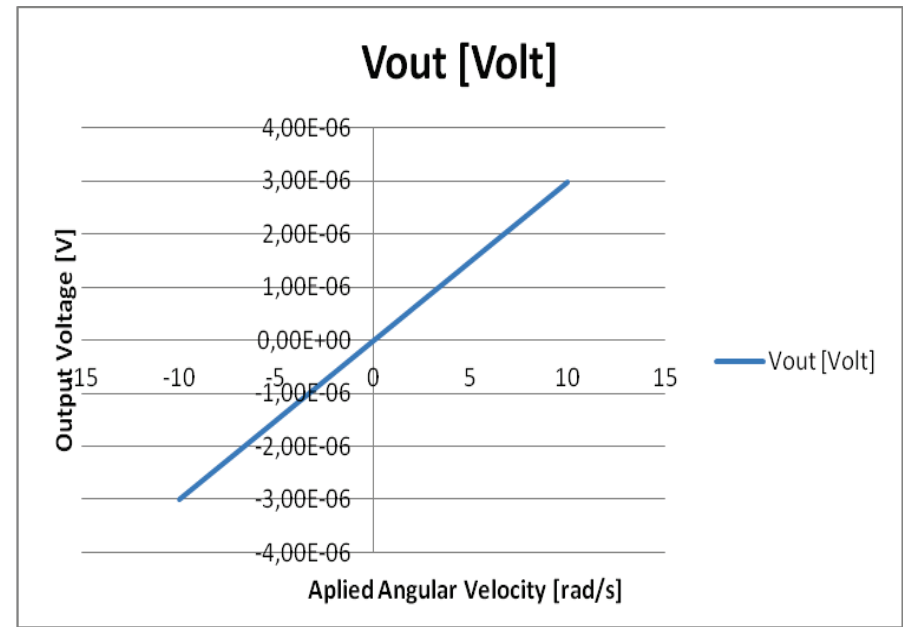

Figure 6. Output voltage vs applied angular velocity for PZT-5H

\section{CONCLUSION}

In this paper, modal analysis was firstly conducted to determine the best operation mode for PMMG and we developed a set of quantitative indicators to evaluate various operation modes. Also, based on the analysis result, it is concluded that the model with integrated masses has better vibration quality of resonance mode are higher sensitivity to the rotation.

\section{REFERENCES}

[1] Soderkvist J. Micromachined Gyroscopes, Sens. Actuators, A43 (1994) $65-71$

[2] Barbour N.M. Inertial Navigation Sensors, (NATO R\&T Organization Publ.) RTO-EN-SET-116 (2011),

[3] Li D.H., Zheng X.J., Wu B., Zhou Y.C. Fracture analysis of a surface through-thickness crack in PZT thin film under a continuous laser irradiation. Engineering Fracture Mechanics 76 (2009) 525-532

[4] Wu X., Chen W., Zhang W., Lu Y., Cui F. Zhao X., Modeling Analysis of Piezoelectric Micromachined Modal Gyroscope. In: Proc. Of the IEEE Intern. Conf. on Nano-Micro Engineered and Molecular Systems (2009, Shenzhen, China)

[5] Lu Y., Wu X., Zhang W., Chen W., Cui F., Liu W., Optimization and analysis of novel piezoelectric solid micro-gyroscope with high resistance to shock, Microsyst. Technol. (2010), 16, 571-584; Optimal special vibration used as reference vibration of vibratory gyroscopes, Electronics Lett., (2010) 46(2)

[6] www.ansys.com

[7] Maenaka K, Kohara H (2006) Novel solid micro-gyroscope. In Proceedings of micro electro mechanical systems workshop (MEMS 2006, Istanbul, Trukey January 2006), pp 634-639

[8] Hu X., Wu X., Wang Z., Chen W., Zhang W., J. Sensors, (2011) ID 106482 